\title{
Paridad y cuotas electorales en Ecuador y México*
}

\author{
Parity and electoral quotas in Ecuador and Mexico
}

Paridade e cotas eleitorais no Equador e México

\author{
Cristiane Aquino Souza*
}

\section{Resumen}

La mayoría de los países de América Latina tienen leyes que establecen cuotas electorales de género. Los efectos de la aplicación de esa legislación cambian de forma significativa entre los diferentes países. Ecuador y México constituyen países donde las cuotas han obtenido éxito, pues han contribuido para un notorio incremento de la participación política femenina en el Parlamento. Este trabajo realiza un análisis del proceso de adopción y eficacia de las leyes que establecieron cuotas electorales de género en Ecuador y México. El objetivo de la investigación ha sido identificar los factores que han influenciado para que la legislación en los mencionados países haya obtenido un alto grado de efectividad en el sentido de aumentar la participación política femenina en los Parlamentos.

Palabras-clave: Cuotas electorales. Paridad. Género. Ecuador y México.

\begin{abstract}
Most Latin American countries have adopted laws establishing electoral gender quotas. The effects of the quota application change significantly between different countries. Ecuador and Mexico are countries where the quotas have
\end{abstract}

* Este trabalho faz parte do projeto de investigação "Democracia e Paridade de gênero", financiado pelo Conselho Nacional de Desenvolvimento Científico e Tecnológico (CNPq) e pela Fundação Cearense de Apoio ao Desenvolvimento Científico e Tecnológico (Funcap).

** Doutora em Direitos Fundamentais pela Universidade Autônoma de Madri (2011), mestra em Direito Público pela Universidade Federal do Ceará (2005), graduada em Direito pela Universidade Federal do Ceará (2002). Atualmente é professora adjunta da Graduação e do Programa de Pós-Graduação em Direito da Universidade de Fortaleza. Fortaleza - CE - Brasil. Email: casfortaleza@hotmail.com 
been successful, because contributed noticeably to the increased participation of women in Parliament. This work analyzes the adoption and the effectiveness of laws that established electoral gender quotas in Ecuador and Mexico. It seeks to identify the factors which influence the high degree of effectiveness reached by these laws to increase women's representation in Parliaments.

Keywords: Electoral quotas. Parity. Gender. Ecuador and Mexico.

\section{Resumo}

A maioria dos países latino-americanos possui leis que estabelecem cotas eleitorais de gênero. Os efeitos da aplicação dessa legislação mudam significativamente entre os diferentes países. Equador e México constituem países onde as cotas obtiveram êxito, pois contribuíram para um notório incremento da participação política feminina no Parlamento. Este trabalho realiza uma análise do processo de adoção e eficácia das leis que estabeleceram cotas eleitorais de gênero no Equador e México. O objetivo da investigação foi identificar os fatores que influenciaram para que a legislação nos mencionados países tenham obtido um alto grau de efetividade no sentido de aumentar a participação política feminina nos Parlamentos.

Palavras-chave: Cotas eleitorais. Paridade. Gênero. Equador e México.

\section{Introducción}

En América Latina varios paísestienen leyes que establecen cuotas de género en las candidaturas electorales ${ }^{1}$. En su conjunto, estos Estados forman dos tercios de las democracias electorales latinoamericanas.Argentina fue el pionero en el mundo en legalizar este mecanismo, al aprobar la "Ley de Cupos" en 1991. La experiencia exitosa de Argentina motivó a otros países de la región a debatir sobre la

\footnotetext{
Para una verificación de los países que adoptan cuotas electorales de género,véase la base de datos del IDEA (Institute for Democracy and Electoral Assistance)/Universidad de Estocolmo. http://www.quotaproject.org/.

2 Hubo una reunión para que las dirigentes argentinas pudieran compartir sus experiencias con mujeres de otros países latinoamericanos celebrada en el Parlamento Latinoamericano de Sao Paulo en 1995.
} 
implementación de cuotas de género ${ }^{2}$. El proceso de redemocratización en América Latina también es señalado como un factor que contribuyó para la implementación de cuotas legales en la región, ya que la adopción de tales medidas es conveniente para conferir una imagen positiva y moderna del país y aumentar la legitimidad del sistema político, en un contexto de reconstrucción de la democracia (ARAÚJO; GARCÍA, 2007, p. 87-88). A esto se suma el importante incentivo proveniente de la IV Conferencia sobre la Mujer de Beijing (1995), cuya Plataforma de Acción busca garantizar el acceso igualitario y la plena participación de las mujeres en las estructuras de poder y toma de decisiones. De esa forma, la mayoría de los países latinoamericanos adoptaron tales leyes en la década de 1990 y más precisamente en 1996 y 1997, años inmediatamente posteriores a la mencionada Conferencia.

Los efectos de la aplicación de cuotas en América Latina cambian de manera significativa entre los países. Aunque es importante resaltar que el alto o bajo porcentaje de mujeres en el parlamento no se debe exclusivamente a la buena o mala efectividad de las cuotas, se puede observar que, pasado cierto tiempo de la existencia de una cuota, si el país logra aumentar el número de mujeres en el parlamento, hay grandes posibilidades de que las cuotas hayan contribuido para ello. Por otro lado, si esto no ocurre, indica que las cuotas no tienen una buena efectividad. Como gran parte de los países latinoamericanos han adoptado cuotas desde la década de 1990, forman los medios ideales para el estudio del impacto de estas medidas. Ecuador y México constituyen ejemplos de países donde las cuotas han contribuido notoriamente para el incremento de la participación de las mujeres en el parlamento. A continuación se va a analizar el proceso de adopción y eficacia de cuotas electorales de género en estos países con el objetivo de identificar los factores que contribuyeron para estos resultados satisfactorios. Es importante resaltar que los aspectos que conciernen a los fundamentos de las cuotas están presentes en otros trabajos de mi autoría. 


\section{Ecuador}

En Ecuador la primera norma sobre cuotas fue prevista en la Ley de Amparo Laboral de 1997, que estableció que la inscripción de listas de candidaturas pluripersonales estaría condicionada a la inclusión de un mínimo del $20 \%$ de candidatas como principales y del $20 \%$ como suplentes. La reforma constitucional de 1998, en el art. 102, abordó la igualdad de género en la participación política, al expresar que

El Estado promoverá y garantizará la participación equitativa de mujeres y hombres como candidatos en los procesos de elección popular, en las instancias de dirección y decisión en el ámbito público, en la administración de justicia, en los organismos de control y en los partidos políticos.

Además, en la disposición transitoria decimoséptima de esta Constitución se reconoció a las mujeres "la participación del veinte por ciento en las listas de elecciones pluripersonales, así como todos los derechos y garantías consagrados en leyes y tratados internacionales vigentes".

En las elecciones de 1998, las mujeres alcanzaron el 17\% de los escaños, lo que representó un gran avance en comparación con el ínfimo 3,6\% conseguido en los comicios de 1996.(UNIÓN INTERPARLAMENTARIA, 2014). La reforma de la Ley de Elecciones, en el año 2000 , aumentó la cuota al $30 \%$ e introdujo un porcentaje progresivo del $5 \%$ para las mujeres en las candidaturas, hasta llegar a la igualdad en la representación:

Las listas de candidaturas en elecciones pluripersonales deberán presentarse con al menos, el treinta por ciento $(30 \%)$ de mujeres entre los principales y el treinta por ciento $(30 \%)$ entre los suplentes; en forma alternada y secuencial, porcentaje que se incrementará en cada proceso electoral general, en un cinco por ciento (5\%) adicional hasta llegar a la igualdad en la representación. Se tomará en cuenta la participación étnica cultural (art. 58 de la Ley General de Elecciones). 
El Tribunal Supremo Electoral redactó el artículo 40 del Reglamento de la Ley General de Elecciones, donde se interpreta que la forma alternada y secuencial permitía ubicar a dos o tres varones por delante de la primera candidata mujer. Esta disposición fue objeto de demanda de inconstitucionalidad ${ }^{3}$ ante el Tribunal Constitucional, que en la Resolución 028-2002-TC, de 12 de noviembre de 2002, consideró el citado artículo inconstitucional, por considerar que

el artículo impugnado realiza una definición de lo que es la alternabilidad y la secuencia, definiciones cuyo contenido no garantizan la participación equitativa entre varones y mujeres; mucho menos cuando al referirse a la secuencia regula la forma en que deben realizarse las combinaciones en serie, atentando a la igualdad de condiciones al establecer que una mujer podría ser ubicada en la papeleta electoral luego de haber sido ubicados dos o tres varones.

Esta decisión del Tribunal Constitucional se pronunció con posterioridad al proceso electoral de 2002, en el que, tras aplicarse un porcentaje mínimo del $35 \%$ de las candidaturas de mujeres, ellas alcanzaron el $16 \%$ de los escaños, porcentaje menor que el conseguido en las elecciones de 1998 (17\%). (UNIÓN INTERPARLAMENTARIA, 2014).No obstante, hay que resaltar que las mujeres constituyeron un $43,5 \%$ del total de candidatos a la diputación (VÁZQUEZ, 2005), lo que resulta un porcentaje significativo de candidatura femenina.

En razón de la decisión del Tribunal Constitucional, en 2004 el Tribunal Supremo Electoral redactó el art. 41 del Reglamento de la Ley de Elecciones en el que estableció un número mínimo de candidatas en conformidad con el número de representantes a elegir y permitió a los partidos definir cómo se concreta la alternancia y la secuencia de mujeres y hombres en la lista ${ }^{4}$.

3 Demanda presentada por la Coordinadora Política de Mujeres Ecuatorianas y la Diputada Anunziatta Valdez Larrea.

$4 \quad$ Art. 41. "La fórmula de representación, de la igualdad de género, en el proceso de inscripción de candidaturas será el siguiente: En elecciones que se elijan tres representantes deberá inscribirse, al menos, una candidata mujer como principal y una como suplente; en elecciones de cuatro a 
En 2008 se aprobó una nueva Constitución en Ecuador, que prevé la paridad entre mujeres y hombres en las directivas partidarias y en las candidaturas a las elecciones pluripersonales, y en este último caso también determina la participación alternada y secuencial de ambos sexos:

Art. 65.- El Estado promoverá la representación paritaria de mujeres y hombres en los cargos de nominación o designación de la función pública, en sus instancias de dirección y decisión, y en los partidos y movimientos políticos. En las candidaturas a las elecciones pluripersonales se respetará su participación alternada y secuencial.

Art. 108.- Los partidos y movimientos políticos son organizaciones públicas no estatales, que constituyen expresiones de la pluralidad política del pueblo y sustentarán concepciones filosóficas, políticas, ideológicas, incluyentes y no discriminatorias.

Su organización, estructura y funcionamiento será democráticos y garantizarán la alternabilidad, rendición de cuentas y conformación paritaria entre mujeres y hombres en sus directivas. Seleccionarán a sus directivas y candidaturas mediante procesos electorales internos o elecciones primarias.

Art. 116.- Para las elecciones pluripersonales, la ley establecerá un sistema electoral conforme a los principios de proporcionalidad, igualdad del voto, equidad, paridad y alternabilidad entre mujeres y hombres; y determinará las circunscripciones electorales dentro y fuera del país.

De esa forma, la Ley Electoral, publicada en abril de 2009, establece que para la selección de candidaturas hay que aplicar "los

\footnotetext{
seis representantes por lo menos, dos serán candidatas mujeres principales y dos suplentes, en elecciones de siete a nueve dignidades, al menos, tres candidatas mujeres como principales y tres como suplentes, siguiendo el procedimiento de alternabilidad anterior; en elecciones de diez a doce representantes, cuatro candidatas mujeres mínimo como principales y suplentes, respectivamente; y, así de manera sucesiva. La alternabilidad y secuencia en la ubicación de puestos de mujeres y hombres será definida por la organización política en el momento de la presentación de la lista, la que contendrá la aceptación de dicha ubicación por parte de todos y cada uno de los candidatos y candidatas".
} 
principios de paridad, alternabilidad, secuencialidad entre los afiliados o simpatizantes de las organizaciones políticas; así como la igualdad en los recursos y oportunidades de candidatos y candidatas" (art. 94). Por otro lado, el art. 99 complementa que "las listas se conformarán paritariamente con secuencia de mujer-hombre u hombre-mujer hasta completar el total de candidaturas principales y suplentes". El art. 105, a su vez, prevé que el Consejo Nacional Electoral y las Juntas Provinciales pueden negar la inscripción de candidaturas cuando "las listas no mantengan de forma estricta la equidad, paridad, alternabilidad y secuencialidad entre mujeres y hombres".

La Constitución y las leyes electorales de Ecuador han puesto énfasis en la alternabilidad y secuencialidad entre mujeres y hombres en las listas, aunque el país adopte listas abiertas. Una de las explicaciones para esto consiste en la posibilidad de votar de manera personalizada o "en plancha" (al partido). Esta segunda opción se lleva a cabo en proporciones considerables, que se modifican en cada elección y que favorecen a las mujeres bien ubicadas en las listas. Así, se defiende la conveniencia de los principios de alternancia y secuencia, "dada esta combinación entre una formal apertura de listas y un voto efectivo «en plancha», en el marco de un sistema político sumamente cambiante y con una normatividad bastante flexible que dificulta prever el comportamiento del electorado"(CAÑETE, 2009, p. 380).

En Ecuador se considera que las cuotas han contribuido a favorecer el incremento progresivo de las mujeres en las listas de candidatura, así como para aumentar el número de diputadas elegidas, aunque no en porcentajes similares (CAÑETE, 2009; VÁZQUEZ, 2005). Los datos indican este análisis, ya que en 1998 el porcentaje de mujeres elegidas fue del $17 \%$; en 2002 , del $16 \%$; en 2006 , del $25 \%$; en 2008 , del $27,63 \%$; en 2009 del $32 \%$ y en 2013, del 38,7\%. (UNIÓN INTERPARLAMENTARIA, 2014).De esa forma, Ecuador ha tenido éxito con la aplicación de las cuotas. Es interesante observar que los sistemas de lista cerrada, bloqueada y con mandatos de posición en favor de la paridad son considerados los mejores para la eficacia de las cuotas 
(HTUN e JONES, 2003; NOHLEN, 1998)5. Ecuador ha conseguido, de hecho, superar este problema. Como ya se ha afirmado, una de las posibles explicaciones para ello es la posibilidad de votar "en plancha" combinada con la alternabilidad y secuencialidad entre mujeres y hombres en la lista.

Por otro lado, se pueden destacar muchos otros factores favorables. Con el progresivo incremento de la cuota, muchas mujeres aumentaron sus expectativas de ser consideradas como candidatas y por eso elaboraron estrategias de presión para favorecer su cumplimiento. También se constató el respaldo político y jurídico a las cuotas, ya que la paridad resultó prevista en la Constitución y el propio presidente (Rafael Correa) se posicionó a favor de la obediencia de la cuota, como también el Poder Judicial, que ha adoptado interpretaciones favorables a la eficacia de la ley. También se observa la existencia de sanciones para el incumplimiento de las cuotas, posibilitando al organismo electoral cumplir su función de control en la inscripción de las listas. Además, el aumento del porcentaje mínimo hasta llegar al 50\% constituye un porcentaje significativo de candidaturas, de modo que los partidos políticos se ven obligados a dedicar mayor apoyo a las campañas de las mujeres, so pena de perder mitad de las posibilidades de obtener escaños. Como menciona Cañete (2009), otro factor que benefició a las candidatas fue la financiación de las franjas electorales por el Estado, que, al asegurar el igual acceso de todas las listas a los medios de comunicación, posibilitaron una mayor visibilización de las mujeres.

\section{México}

En México se implementaron diversas reformas en la legislación electoral para favorecer la participación política de las mujeres. En 1993 se alteró la fracción 3 del art. 175 del Código Federal de Instituciones y Procedimientos Electorales (COFIPE), para recomendar a los partidos

5 Ese consenso ha sido cuestionado por algunos académicos (SCHMIDT; SAUNDERS, 2004). 
políticos el incentivo de una mayor participación política femenina: "Los partidos políticos promoverán, en los términos que determinen sus documentos internos, una mayor participación de las mujeres en la vida política del país, a través de su postulación a cargos de elección popular". En 1996 la legislación exhortaba a los partidos a establecer una cuota máxima del $70 \%$ del mismo género en las candidaturas electorales: "Los partidos políticos nacionales considerarán en sus estatutos que las candidaturas por ambos principios a diputados y senadores no excedan el 70 por ciento para un mismo género. Asimismo promoverán la mayor participación política de las mujeres." (Transitorio XXII del COFIPE).

Sin embargo, la norma no preveía mandato de posición ni sanciones por incumplimiento. De ahí que las mujeres eran ubicadas en posiciones de las listas con nula posibilidad de elección o eran puestas como suplentes (BARQUET; CERVA, 2005). De esa forma, en las elecciones de 1997, el incremento de diputadas fue muy pequeño (tres puntos porcentuales) y en las de 2000 ha incluso disminuido de $17,4 \%$ a $16,8 \%$ (TRIBUNAL ELECTORAL DEL PODER JUDICIAL DE LA FEDERACIÓN, 2014).

En 2002 otra reforma del Código Federal de Instituciones y Procedimientos Electorales buscó perfeccionar la norma, cuando se determinó la cuota mínima del $30 \%$ para candidaturas a diputados y senadores juntamente con un mandato de posición de al menos un candidato de género distinto a cada segmento de tres candidaturas:

Art. 175-A De la totalidad de solicitudes de registro, tanto de las candidaturas a diputados como de senadores que presenten los partidos políticos o las coaliciones ante el Instituto Federal Electoral, en ningún caso incluirán más del setenta por ciento de candidatos propietarios de un mismo género.

Art. 175-B Las listas de representación proporcional se integrarán por segmentos de tres candidaturas. En cada uno de los tres primeros segmentos de cada lista habrá una candidatura de género distinto. Lo anterior sin perjuicio de los mayores avances que en esta materia señale la 
normatividad interna y los procedimientos de cada partido político.

Es relevante destacar la singularidad del sistema electoral mexicano para la elección de los miembros de la Cámara Baja. Los 500 diputados son elegidos por un sistema electoral mixto. Así, 200 son elegidos en 5 distritos plurinominales a través de representación proporcional y 300 provienen de distritos uninominales de mayoría relativa. La legislación preveía que un máximo de 210 candidaturas, sobre un total de 300 distritos uninominales de mayoría relativa, podían pertenecer a un mismo género. No obstante, como ya se ha observado, los distritos uninominales dificultan la aplicación de las cuotas (REYNOSO, 2008) y no favorecen la elección de las mujeres (FAVELA, 2010). Así, la existencia de distritos uninominales parece ser un factor perjudicial a una mayor eficacia de las cuotas en las elecciones a la Cámara Baja en México. Por otro lado, la ley impone sanciones por su incumplimiento, que consisten en amonestación pública y, si necesario, posterior negativa de registro de las candidaturas:

\section{Art. 175-C}

1. Hecho el cierre del registro de candidaturas, si un partido político o coalición no cumple con lo establecido en los artículos 175-A y 175-B, el Consejo General del Instituto Federal Electoral le requerirá en primera instancia para que en el plazo de 48 horas, contadas a partir de la notificación, rectifique la solicitud de registro de candidaturas y le apercibirá de que, en caso de no hacerlo le hará una amonestación pública.

2. Transcurrido el plazo a que se refiere el párrafo anterior, el partido político o coalición que no realice la sustitución de candidatos, será acreedor a una amonestación pública y el Consejo General del Instituto Federal Electoral le requerirá, de nueva cuenta, para que en un plazo de 24 horas, contadas a partir de la notificación, haga la corrección. En caso de reincidencia se sancionará con la negativa del registro de las candidaturas correspondientes. 
La ley establece también una norma que es considerada una cláusula de escape que le posibilita a los partidos no observar la cuota. El art. 175-C "3" del Código Federal de Instituciones y Procedimientos Electorales excluye de sanción a "las candidaturas de mayoría relativa que sean resultado de un proceso de elección mediante voto directo". Así, los partidos podían realizar elecciones primarias para evitar cumplir con la cuota de género. En efecto, la opción por las primarias ocurrió en varios $\operatorname{casos}^{6}$, hecho que mitigó el efecto de las cuotas. Aun así, en las elecciones del 2003 el porcentaje de diputadas ascendió de un 16,8\% a un $24,9 \%$. Sin embargo, en las elecciones de 2006 descendió otra vez al $22,6 \%$.

En 2007 se realizó otra Reforma Electoral Federal en México y el nuevo Código Federal de Instituciones y Procedimientos Electorales ha sido publicado en enero de 2008. En lo que concierne a la igualdad de género en la participación política, se ha aumentado el porcentaje mínimo de candidatura a diputados o senadores para un mismo género, que pasó a ser del $40 \%$, procurando llegar a la paridad:

De la totalidad de solicitudes de registro, tanto de las candidaturas a diputados como de senadores que presenten los partidos políticos o las coaliciones ante el Instituto Federal Electoral, deberán integrarse con al menos el cuarenta por ciento de candidatos propietarios de un mismo género, procurando llegar a la paridad. (Art. 219.1, COFIPE)

El porcentaje mínimo incide sobre los candidatos propietarios, evitando la posibilidad de cumplir con la cuota mediante la ubicación de mujeres en las listas de suplencia.

6 En las elecciones para los 300 distritos uninominales, el PRD realizó primarias para el 15\% de ellos en 2003 y para un $27 \%$ en 2006. El PAN efectuó primarias para un $52 \%$ en 2003 y un $53 \%$ en 2006 y por fin, el PRI realizó primarias en un $98 \%$ en 2003, pero ninguna en el 2006 . Según Lisa Baldez(2008, p. 170-172), "las votaciones directas efectuadas, en muchos de los casos, no fueron realmente tan directas; más bien constituyeron votaciones nominales para ratificar los nombramientos ya efectuados por la cúpula partidista". 
Se han mantenido las mismas sanciones. Las reglas sobre el mandato de posición han sido modificadas. El art. 220 del COFIPE menciona que "Las listas de representación proporcional se integrarán por segmentos de cinco candidaturas. En cada uno de los segmentos de cada lista habrá dos candidaturas de género distinto, de manera alternada".

Esta norma posibilita varias interpretaciones sobre el significado concreto de la regla de alternancia por segmentos (RUIZ MIGUEL, 2009). En el caso Mary Telma Guajardo Villarreal Vs. Comisión de Garantías del Partido de la Revolución Democrática ${ }^{7}$ el Tribunal Electoral del Poder Judicial de la Federación (TEPJF) consideró que la regla de alternancia consiste en "colocar en forma sucesiva una mujer seguida de un hombre, o viceversa, en cada segmento de cinco candidaturas hasta agotar dicho número, de modo tal que el mismo género no se encuentre en dos lugares consecutivos del segmento respectivo".

Con la reforma de 2007, el COFIPE establece también en el art. 38.s la obligación de los partidos de "garantizar la equidad y procurar la paridad de los géneros en sus órganos de dirección y en las candidaturas a cargos de elección popular". En el mismo sentido, el art. 218.3 dispone que los partidos

promoverán y garantizarán en los términos del presente ordenamiento, la igualdad de oportunidades y procurarán la paridad de género en la vida política del país, a través de postulaciones a cargos de elección popular en el Congreso de la Unión, tanto de mayoría relativa como de representación proporcional.

No hay que obviar que en el art. 78.V, del COFIPE se reguló que cada partido deberá destinar anualmente, el $2 \%$ del financiación pública

\footnotetext{
Sentencia SUP-JDC-461/2009. En este caso, la autora logró su pretensión de ser trasladada del lugar cuatro a la posición tres de la lista de candidatos a diputados. En la lista impugnada el partido había colocado a una mujer, seguida de dos hombres y luego de dos mujeres, mientras que la demandante alegó que la regla de alternancia obliga a intercalar, de manera sucesiva, a un hombre y a una mujer entre sí.
} 
ordinaria a la capacitación, promoción y desarrollo del liderazgo político de las mujeres. En las elecciones de 2009, celebradas después de la mencionada reforma, el porcentaje de diputadas elegidas aumentó del 22,6\% al 28,2\%(UNIÓN INTERPARLAMENTARIA, 2014), Ilegando así a cerca del $30 \%$, aunque todavía sin alcanzar el $40 \%$. No obstante, ciertas prácticas todavía impiden el logro de una igualdad efectiva. El famoso caso de las denominadas "juanitas" pone en evidencia la maniobra de postular a las mujeres como candidatas propietarias para que posteriormente renuncien con la intención de ser sustituidas por el hombre suplente. El mencionado caso se refiere a ocho mujeres que lograron escaños de diputada federal en las elecciones de 2009 y que a principios del mes de septiembre solicitaron licencia para separarse del cargo y ceder espacio a su suplente, que en todos los casos era un hombre. Tras la discusión sobre la viabilidad o no de conceder las licencias, a siete de las diputadas se les confirió una respuesta afirmativa. Posteriormente, ocho diputadas más han sido sustituidas por sus suplentes hombres, de modo que hasta febrero de 2010 un total de quince hombres han asumido el cargo de diputados federales en razón de la suplencia de mujeres ${ }^{8}$ que renunciaron (FAVELA, 2010). Así, en marzo de 2010 el porcentaje de diputadas disminuyó al 25,8\%. Eso demuestra la existencia de casos en que los partidos buscan fórmulas para burlar las cuotas y que a veces lo hacen con la complicidad de algunas mujeres.

Es necesario resaltar también que en esa Reforma de 2007 el COFIPE establece, de forma similar a la norma anteriormente existente, que están exceptuadas de aplicar las cuotas "las candidaturas de mayoría relativa que sean resultado de un proceso de elección democrático, conforme a los estatutos de cada partido". Dicha regla fue materia del Acuerdo CG327/2011, del Consejo General del Instituto Federal Electoral (CGIFE), de siete de octubre de 2011, cuyo precepto decimotercero establece que se debe entender por procedimiento democrático "aquel

Por otra parte, en este mismo espacio de tiempo solamente cuatro hombres solicitaron licencia en beneficio de suplentes mujeres. 
en el que la elección de las candidaturas se realice de forma directa por la militancia del partido o por la ciudadanía, o de forma indirecta a través de una convención o asamblea en que participe un número importante de delegados electos ex profeso por dicha militancia”. El artículo 219, párrafo 2 del COFIPE, sumado a la interpretación dada por el CGIFE en el Acuerdo CG327/2011, constituyó un obstáculo a la paridad. Eso porque los partidos políticos podían seguir realizando elecciones primarias para evitar cumplir con la cuota de género.

En razón de esto, dicho acuerdo ha sido impugnado ante el TEPJF, que en la sentencia SUP-JDC-12624/2011, ha determinado expulsar de su punto decimotercero la parte que explica lo que se debe entender por procedimiento democrático. Por otro lado, ha determinado la modificación de ese acuerdo, en el sentido de establecer que, aun cuando el partido político o coalición elija a sus candidatos de mayoría relativa mediante un proceso de elección democrático, "deberá presentar como mínimo 120 y 26 candidatos propietarios de un mismo género, a Diputados y Senadores, respectivamente".

EI TEPJF, en la mencionada sentencia, considera que el párrafo cuarto del punto decimo tercero del acuerdo impugnado viola el principio de jerarquía normativa en la medida en que modifica el contenido de la fracción 2 del artículo 219 del COFIPE. Por otro lado, entiende que los procedimientos para la designación de candidatos previstos en los estatutos vigentes de los partidos se presumen constitucionales y legales y, por lo tanto, democráticos. Todos los estatutos vigentes, por consecuencia, serían adecuados para hacer efectiva la excepción prevista en el párrafo 2 del artículo 219 del Código de la materia, lo que haría nugatorio las cuotas de género (SUP-JDC-12624/2011).

La importante fundamentación de la mencionada Sentencia consiste en considerar que el principio del respeto a la cuota de género requiere una interpretación con perspectiva de género. Eso porque el artículo 1, párrafo 2 de la Constitución Federal dispone que las normas relativas a los derechos humanos se deben interpretar favoreciendo en todo tiempo, a las personas, la protección más amplia. Así que 
la limitación a las normas de igualdad de género, en el contexto de registro de candidaturas a diputados y senadores por el principio de mayoría relativa debe ser restringida a su más mínima dimensión, "de tal manera que no se encuadren en la misma más supuestos que los mínimos para no hacer nugatorio en la realidad ese tipo de derechos" (SUP-JDC-12624/2011). De esa forma, el CGIFE no hizo, según el Tribunal, una interpretación conforme del artículo 219 del COFIPE. Así, como se ha observado, el TEPJF no sólo pide que se expulse del acuerdo impugnado la definición de procedimiento democrático, pero también establece que, aun cuando el partido utilice procedimientos democráticos, deberá aplicar las cuotas, presentando como mínimo 120 y 26 candidatos propietarios de un mismo género.

Esta sentencia es parte de un conjunto de decisiones del Poder Judicial Federal que adoptan una interpretación en el sentido de posibilitar la eficacia de las cuotas. Está claro que la posibilidad de los partidos de recurrir a elecciones internas sin aplicar las cuotas limita su eficacia de manera significativa. Se puede argumentar, en contrapartida, que fue el propio legislador quien hizo la opción de privilegiar las elecciones internas democráticas. Sin embargo, también ha sido el propio legislador quien, en inúmeras otras normas, ha buscado conferir un espacio de relevancia a la paridad.

De esta forma, estas normas deben ser interpretadas en conjunto. Y, como bien ha afirmado el Tribunal, una vez que se trata de una norma relativa a los derechos humanos, se deben comprender favoreciendo en todo tiempo, a las personas, la protección más amplia (SUPJDC-12624/2011). Este mismo raciocinio se aplica a la determinación de la Sentencia SUP-JDC-12624/2011, al señalar que el acuerdo impugnado debe ser modificado para establecer también que, en las listas de representación proporcional, la fórmula completa (propietario y suplente) debe integrarse por candidatos del mismo género (SUPJDC-12624/2011). Esta decisión ciertamente favorece la eficacia de la paridad, al impedir que los partidos adopten fórmulas para burlar las cuotas, como ocurrió en el caso de las denominadas "juanitas". 
Por fin, atendiendo a la ejecutoria mencionada, el CGIFE adoptó las modificaciones requeridas mediante el Acuerdo CG413/2011, de 14 de diciembre de 2011. Los datos estadísticos indican que esta forma de interpretar y aplicar la ley de cuotas contribuye a su eficacia, ya que el número de mujeres en la Cámara Baja de México pasó del 28,2\% en el 2009 al 36,80\%en las elecciones del 2012, es decir, se alcanzó un porcentaje mucho más cercano a la paridad. En el Senado el porcentaje de mujeres electas aumentó del 17,9\% en las elecciones de 2006 al $32,81 \%$ en las elecciones de 2012.

Ante las evidencias comentadas, parece que México constituye un país en el que las cuotas han tenido éxito.Uno de los factores que contribuyen a la eficacia del mecanismo son las listas cerradas y bloqueadas en el sistema proporcional ${ }^{9}$. Por otro lado, la norma se ha mejorado posteriormente, con la previsión del mandato de posición y de sanciones legales para su incumplimiento. También se debe resaltar la exigencia de aplicación de la ley por parte de ciertas mujeres políticas. Un factor que se destaca para el logro del resultado alcanzado en México es las varias interpretaciones favorables a la eficacia de las cuotas por parte del Tribunal Electoral del Poder Judicial de la Federación.

\section{Conclusión}

Ecuador y México construyen países donde las cuotas han tenido un gran éxito en el sentido de aumentar la participación femenina en el Parlamento. Ante el estudio realizado, uno de los factores que contribuyen para el resultado alcanzado es, en el caso de México, es la previsión de listas cerradas y bloqueadas en el sistema proporcional combinado con

9 Una investigación desarrollada por la Unión Europea en 1997 señala la superioridad del sistema de representación proporcional sobre el mayoritario en lo que concierne a la representación política de las mujeres. El estudio resalta que los países con niveles más bajos de representación femenina tienen un sistema mayoritario (Reino Unido y Francia) o un sistema mixto de representación proporcional débil (Italia y Grecia). Por otra parte, los Estados con un alto porcentaje de mujeres representantes tienen un sistema de representación proporcional o mixto. (UNIÓN EUROPEA, 1997). 
el mandato de posición. En el caso de Ecuador, el supuesto obstáculo de las listas abiertas es superado por medio de la posibilidad de votar "en plancha" sumada a la alternabilidad y secuencialidad entre mujeres y hombres en las listas.

Otro factor relevante en los dos países es la previsión legal de sanciones para el incumplimiento de la norma. Se ha observado una gran resistencia de los partidos en aplicar las cuotas y en poner a las mujeres en posiciones posibles de resultas electas. Por ello, es importante la existencia de sanciones y de un organismo electoral que imponga a los partidos la obediencia a la legislación, como se ha constatado en los países investigados.

Un factor destacable en México y Ecuador son las interpretaciones favorables a la eficacia de las cuotas por parte del Poder Judicial. En México, por ejemplo, el entendimiento de que la paridad debe ser aplicada aún cuando en partido político elija a sus candidatos de mayoría relativa mediante un proceso de elección democrático ha sido determinante para el aumento del número de mujeres electas.

No hay que obviar que en Ecuador y México la previsión de cuotas electorales legales han evolucionado hacia la adopción de la paridad electoral $^{10}$. Con el incremento de las cuotas hasta llegar al $50 \%$ en Ecuador y $40 \%$ en México, los partidos políticos se ven obligados a dedicar mayor apoyo a las campañas de las mujeres, so pena de perder gran parte de las posibilidades de obtener escaños. El porcentaje de mujeres electas en las últimas elecciones en estos países para la Cámara

10 El porcentaje del $40 \%$, aunque no corresponda a una paridad estricta, es comprendido como un valor que promueve la participación equilibrada entre mujeres y hombres, lo que se puede entender como paridad en sentido amplio. La Resolución del Parlamento Europeo, de 18 de enero de 2001, sobre el informe de la Comisión respecto a la aplicación de la Recomendación 96/694 del Consejo, de 2 de diciembre de 1996, exige que se favorezca el equilibrio de mujeres y hombres en todas las políticas y en todas las comisiones, tanto en la Unión Europea, como a nivel internacional e internacional, con una participación que no debe ser inferior al $40 \%$ para cada género.En el mismo sentido se pronuncia la Recomendación Rec (2003)3 del Comité de Ministros del Consejo de Europa sobre la participación equilibrada de mujeres y hombres en los procesos de toma de decisión en los ámbitos político y público. 
Baja en México, en el año 2012 (36,80\%) y en Ecuador ${ }^{11}$, enel año $2013(38,69 \%)$ y en la Cámara Alta de México en el año 2012 (34,38\%) está próximo al 40\%. De esa forma, estos países constituyen ejemplos para los demás en la búsqueda de un mayor equilibrio entre mujeres y hombres en la política y, más específicamente, en el Parlamento.

\section{Referencias}

ARAÚJO, Clara; GARCÍA, Ana Isabel. The experience and the impact of quotas in Latin America. In: DAHLERUP, Drude (Org.). Women, quotas and politics. New York: Routledge, 2007. p. 83-111.

BALDEZ, Lisa. Cuotas versus primarias: La nominación de candidatas mujeres en México. In: RÍOS TOBAR, Marcela (Org.). Mujer y política: el impacto de las cuotas de género en América Latina. Santiago: Catalonia, 2008. p.157-177.

BARQUET, Mercedes; CERVA, Daniela. La lente percée des femmes au Congrès. In: TREMBLAY, Manon (Org.). Femmes et parlements: un regard international. Montréal: Remue-ménage, 2005. p. 457-474.

CAÑETE, María Fernanda. Una paridad formal que no se traduce en paridad real: la cuota y la participación electoral de las mujeres ecuatorianas. In: BAREIRO, Line; TORRES, Isabel (Org.). Igualdad para una democracia incluyente. San José: IIDH, 2009. p. 363-382.

FAVELA, Adriana. Comentarios a la obra género y derechos políticos: la protección jurisdiccional de los derechos político-electorales de las mujeres en México. In: SEMINARIO JUSTICIA ELECTORAL Y EQUIDAD DE GÉNERO, 2010. México, DF : Tribunal Electoral del Poder Judicial de la Federación. 2010. Disponible en: <http://www.te.gob.mx/ Justicia_Electoral/pdf/genero_derechos_politicos.pdf >. Acceso en: 14 mayo 2014 .

11 Ecuador adopta el sistema unicameral. 
HTUN, MALA; JONES, Mark. Engendering the right to participate in decision-making: electoral quotas and women's leadership in Latin America. In: CRASKE, Nikki; MOLYNEUX, Maxine (Org.). Gender and the politics of rights and democracy in Latin America. Londres: Palgrave, 2003. p. 32-57.

NOHLEN, Dieter. América Latina: Una comparación de sus sistemas electorales. In: NOHLEN, Dieter, PICADO, Sonia; ZOVATTO, Daniel (Org.). Tratado de derecho electoral comparado de América Latina. México, DF: Fondo de Cultura Económica, 1998, p. 43-70.

REYNOSO, Diego. El exiguo impacto de las leyes de cuotas en México. In: ARCHENTI, Nélida; TULA, María Inés (Org.). Mujeres y política en América Latina: sistemas electorales y cuotas de género. Buenos Aires: Heliasta, 2008. p. 107-135.

RUIZ MIGUEL, Alfonso. Igualdad, libertad y unidad política: a propósito de las cuotas electorales en México. SEMINARIO INTERNACIONAL DEL OBSERVATORIO JUDICIAL ELECTORAL, TRIBUNAL ELECTORAL DEL PODER JUDICIAL DE LA FEDERACIÓN, 2., 2009, México, DF. Anais... México, DF: [s.n], 2009. Disponible en: <http://www.te.gob. $\mathrm{mx} / \mathrm{ccje} /$ Archivos/ponencias/alfonso_ruiz_miguel.pdf >. Acceso en: 15 mayo 2014.

SCHMIDT, Gregory D; SAUNDERS, Kyle L. Effective quotas, relative party magnitude, and the success of female candidates: peruvian municipal elections in comparative perspective. Comparative Political Studies, Thousand Oaks, v.37, n.6, p. 704-34, 2004.

TRIBUNAL ELECTORAL DEL PODER JUDICIAL DE LA FEDERACIÓN. Disponivel em: <http://www.trife.gob.mx/sitemap>. Acesso em: 15 mayo 2014.

UNIÓN EUROPEA. Impacto diferencial de los sistemas electorales en la representación política femenina. Dirección General de Estudios. Documento de Trabajo. Serie Derechos de la Mujer, W-10, 1997. Disponível em: <http://www.europarl.europa.eu/workingpapers/ femm/w10/1_es.htm>. Acesso em: 14 maio 2014. 
UNIÓN INTERPARLAMENTARIA. Disponível em: <http://www.ipu.org/ parline-e/parlinesearch.asp>. Acesso em: 14 maio 2014.

VÁZQUEZ, Luz Entrena. Constitución y acciones positivas: el sistema de cuotas de participación política para mujeres en Ecuador. In: ANUARIO de Derecho Constitucional Latinoamericano. Montevideo: Konrad Adenauer Stiftung, 2005. v. 2. p. 501-514.

Recebido em: 19/05/14

Aprovado em: 07/10/14 